\title{
Serum Levels of Natriuretic Peptides in Children before and after Treatment for an Atrial Septal Defect, a Patent Ductus Arteriosus, and a Coarctation of the Aorta-A Prospective Study
}

\author{
Anneli Eerola,, ${ }^{1,}$ Eero Jokinen, ${ }^{2}$ Talvikki Boldt, ${ }^{2}$ Ilkka P. Mattila, ${ }^{3}$ and Jaana I. Pihkala ${ }^{2}$ \\ ${ }^{1}$ Department of Pediatrics, University Hospital of Tampere, 33521 Tampere, Finland \\ ${ }^{2}$ Department of Pediatric Cardiology, Hospital for Children and Adolescents, University of Helsinki, Helsinki, Finland \\ ${ }^{3}$ Department of Pediatric Cardiac Surgery, Hospital for Children and Adolescents, University of Helsinki, Helsinki, Finland
}

Correspondence should be addressed to Anneli Eerola, anneli.eerola@fimnet.fi

Received 27 September 2009; Revised 13 December 2009; Accepted 8 February 2010

Academic Editor: Amiram Nir

Copyright () 2010 Anneli Eerola et al. This is an open access article distributed under the Creative Commons Attribution License, which permits unrestricted use, distribution, and reproduction in any medium, provided the original work is properly cited.

\begin{abstract}
Background and Objectives. We evaluated and compared the influence of treatment for atrial septal defect (ASD), patent ductus arteriosus (PDA), and coarctation of the aorta (CoA) on serum levels of N-terminal proatriopeptide and N-terminal probrain natriuretic peptide. Correlations between peptide levels and echocardiographic measurements were calculated. Patients and Methods. Peptide levels were measured and echocardiography performed before and 6-12 months after treatment in 21 children with ASD, 25 with PDA, 15 with CoA, and 76 control children. Results. ANPN levels were higher than in controls at baseline in all patient groups, and NT-proBNP in patients with ASD and PDA. Both peptide levels were elevated 6 months after treatment and decreased thereafter. Peptide levels were higher in patients with volume than pressure overload. They correlated with echocardiographic measurements. At the 6-month follow-up, dimensions of the originally overloaded ventricle had normalized only in patients with PDA. Conclusions. After intervention, peptide levels decrease but normalization takes over 6 months. The type of correlation between peptide levels and echocardiography varies according to the loading condition. Measurement of peptide levels can be used for monitoring the course of a patient's heart disease.
\end{abstract}

\section{Introduction}

Atrial natriuretic peptide (ANP), brain natriuretic peptide (BNP), C-type natriuretic peptide, and dendroaspis natriuretic peptide form the natriuretic peptide system. They have an important role in regulation of fluid homeostasis, blood pressure/vascular tone, and cardiomyocyte growth. This system functions to retard the progression of heart failure, opposite to the effect of neurohumoral systems like the reninangiotensin-aldosterone system and the sympathetic nervous system $[1,2]$.

ANP is produced primarily in the atrial myocyte and in smaller amounts in ventricular myocytes. It appears in fetal ventricular tissue and in hypertrophied ventricles. It is stored as a prohormone in atrial granules and released in response to wall stress. In the presence of heart disease, ANP gene expression is upregulated in ventricular myocytes
$[2,3]$. BNP is produced in atrial and ventricular myocytes. In healthy people, the main site of gene expression is atrial, but in disease states such as heart failure, ventricular gene expression is upregulated $[4,5]$. BNP is stored together with ANP in storage granules in the atrial myocytes in the healthy state. The stimulus for synthesis and release of BNP is wall stress $[2,3]$.

Normal levels of ANP and BNP and their inactive Nterminal fragment have been investigated using different methods [6-11]. For each method, a specific range of normal values exits. The levels of natriuretic peptides have been shown to be higher immediately after birth and to decrease thereafter, during the first 2-4 months [10-12]. Studies on levels of natriuretic peptides in pediatric patients with different types of congenital heart defects and heart failure have been published. Unfortunately, the follow-up time in most has been short, and study groups have consisted of 
TABLE 1: Characteristics of subjects in the study groups at baseline. Values are expressed as median (range).

\begin{tabular}{lccccccc}
\hline Group & $N$ & Gender $(\mathrm{m} / \mathrm{f})$ & Age (years) & No. of patients aged less than 6 months & Weight $(\mathrm{kg})$ & Height $(\mathrm{cm})$ & BSA $\left(\mathrm{m}^{2}\right)$ \\
\hline $\begin{array}{l}\text { Controls } \\
\text { T6 }\end{array}$ & $32 / 44$ & $\begin{array}{c}5.4 \\
(0.0-15.6)\end{array}$ & 12 & $\begin{array}{c}20.4 \\
(3.1-66.5)\end{array}$ & $\begin{array}{c}113.8 \\
(48.0-174.6)\end{array}$ & $(0.19-1.81)$ \\
\hline $\begin{array}{l}\text { Patients } \\
\text { with ASD }\end{array}$ & 21 & $7 / 14$ & $\begin{array}{c}5.2 \\
(2.3-14.9)\end{array}$ & 0 & 20.0 & $\begin{array}{c}(12.4-59.0) \\
(83.0-163.0)\end{array}$ & $(0.53-1.63)$ \\
\hline $\begin{array}{l}\text { Patients } \\
\text { with PDA }\end{array}$ & 25 & $11 / 14$ & $\begin{array}{c}2.6 \\
(1.0-10.6)\end{array}$ & 0 & 13.0 & 87.5 & 0.54 \\
$\begin{array}{l}\text { Patients } \\
\text { with CoA }\end{array}$ & 15 & $8 / 7$ & 2.0 & 7 & $16.9-32.8)$ & $(70.0-139.5)$ & $(0.36-1.14)$ \\
\hline
\end{tabular}

ASD: atrial septal defect; BSA: body surface area; CoA: coarctation of the aorta;

PDA: patent ductus arteriosus.

different types of congenital heart defects [13-16]. To our knowledge, comparison of the effect of treatment for various types of loading conditions on levels of natriuretic peptides has not been performed.

Atrial septal defect (ASD) predisposes the patient to volume overload of the right side of the heart, increased pulmonary vascular resistance, and atrial arrhythmias [17, 18]. The physiological sequelae of ASD depend on the size of the defect, the relation of the diastolic compliances of the right (RV) and left ventricle (LV), and the ratio of pulmonary to systemic vascular resistance [17]. Patent ductus arteriosus (PDA) causes volume overload of the left side of the heart [19] and predisposes the patient to pulmonary hypertension. Coarctation of the aorta (CoA) causes pressure overload of the LV and predisposes the patient to systemic hypertension [20], which may lead to myocardial hypertrophy and diastolic and systolic dysfunction [21].

The timing of treatment for congenital heart defect is based on the hemodynamic and anatomic situation, with consideration of myocardial cell adaptation and chamber remodeling. Therefore, it is important to have multiple methods available for follow-up. The combination of new imaging modalities and measurement of serum levels of natriuretic peptides may allow us to improve evaluation of cardiac function and timing of interventions.

$\mathrm{N}$-terminal proatriopeptide (ANPN) and N-terminal probrain natriuretic peptide (NT-proBNP) may have different regulation of gene expression depending on the form and localization of the stress exerted on the heart. Therefore, treatment for different types of loading condition may cause different types of changes in levels of these peptides. The purpose of this study was to evaluate the influence of treatment for different loading conditions on serum levels of ANPN and NT-proBNP. We correlated peptide levels to two-dimensional (2D) and three -dimensional (3D) echocardiographic findings in these conditions.

\section{Methods}

This prospective follow-up study was carried out at the Hospital for Children and Adolescents, University of Helsinki, Helsinki, Finland, between February 2003 and February 2006. All parents agreed to have their children participate in this clinical trial approved by the hospital ethics committee and gave their written informed consent.

The study groups consisted of 61 patients undergoing percutaneous or surgical treatment for ASD, PDA, or CoA. Twenty-one patients had an ASD, 25 a PDA, and 15 a CoA. The control group comprised 76 healthy voluntary children of whom 64 were 6 months or older. Demographics of children with ASD, PDA, CoA, and controls are shown in Table 1.

All patients and controls underwent clinical cardiovascular examination and blood test sampling for measurement of natriuretic peptides at the time of echocardiographic examinations. All patients were examined just prior to the intervention and 6 months later. The children with ASD and CoA were also examined 12 months after intervention. Control children were examined once. They were asymptomatic and showed no abnormalities in clinical examination, ECG, or echocardiography.

Serum samples were frozen at $-20^{\circ} \mathrm{C}$. Serum concentrations of ANPN were measured by immunofluorometric assay. The reagents were manufactured by Medix Biochemica (Espoo, Finland) and the instruments by Delfia Research Fluorometer (Wallac, Turku, Finland). Serum concentrations of NT-proBNP were measured by the electrochemiluminometric method. The reagent kit was manufactured by Roche (Mannheim, Germany), and the samples were analyzed at Limbach Laboratory (Heidelberg, Germany).

2.1. Echocardiography. We used the Acuson Sequoia C256 echocardiography system (Siemens, Mountain View, CA) for 2D imaging and for Doppler and M-mode measurements. Three-dimensional echocardiography was performed with TomTec computer software (TomTec Imaging Systems GmHb, Munich, Germany). Two-dimensional echocardiographic examinations were carried out by two observers (A. Eerola and E. Jokinen). All 3D examinations and the analyses of echocardiographic measurements were carried out by one observer (A. Eerola).

2.2. 2D Echocardiography. Two-dimensional echocardiography with color flow Doppler was used to evaluate the cardiac anatomy and to measure the size of ASD. M-mode echocardiography was performed from either the parasternal 
long-axis or short-axis views. RV end-diastolic and LV enddiastolic and end-systolic dimensions were measured, and LV volumes, fractional shortening, and ejection fraction calculated. The $z$-scores of thicknesses of interventricular septum and posterior wall of LV, and those of RV and LV end-diastolic dimensions were determined [22]. The mean of measurements from three cardiac cycles for each participant was saved for analysis.

2.3. 3D Echocardiography. Three-dimensional echocardiography was performed with TomTec computer software system, as described earlier in [23]. The time-volume curves obtained served to determine end-diastolic and end-systolic volumes, stroke volume, and ejection fraction.

2.4. Cardiac Catheterization. Cardiac catheterization with hemodynamic evaluation and angiography was performed under general endotracheal anesthesia, systemic heparinization, and antimicrobial prophylaxis. In patients with ASD, the procedure was also guided by transesophageal echocardiography. For ASD closure, either the Amplatzer Septal Occluder (AGA Medical Corp., Golden Valley, MN) or the Helex Septal Occluder (W.L. Gore and Associates, Flagstaff, AZ) was used. The PDA was occluded with either Flipper detachable coil (Cook, Bloomington, IN) or with an Amplatzer Duct Occluder (AGA Medical Corp.). In patients with CoA, percutaneous angioplasty was performed using either balloon dilatation only or with stent implantation.

2.5. Surgery. Surgical closure of ASD was performed via midline sternotomy with direct suture using normothermic cardiopulmonary bypass. Repair of CoA was performed with resection and end-to-end anastomosis from left thoracotomy.

\section{Statistical Analysis}

Analyses were performed with the Statistical Package for Social Science version 12.01 for Windows (SPSS Inc., Chicago, IL). For variables derived from blood samples, median and range were calculated. Because distribution of parameters tested by Kolmogorov-Smirnov's goodness-of-fit test was not normal, the Mann-Whitney test was used for statistical analysis between groups, and the Wilcoxon SignedRank Test for analysis within groups. Spearman's correlation coefficient was used for calculating correlations between the echocardiographic measurements of chamber dimensions and serum concentrations of ANPN and NT-proBNP. The level of significance was $P<.05$.

\section{Results}

4.1. Children with ASD. Of the 21 children with ASD, 14 were treated with device closure and 7 with operation. The size of the defect measured a median of 12 (range 7-21) mm in transthoracic echocardiography. No other heart defects were observed. The ASD was closed with the Amplatzer Septal Occluder (AGA Medical Corp., Golden Valley, MN) in
12 patients and with the Helex Septal Occluder (W. L. Gore and Associates, Flagstaff, AZ) in 2 patients. After closure, no residual shunt was evident at the 6- or 12-month follow-up in transthoracic echocardiography.

At baseline, children with ASD had higher serum levels of both ANPN and NT-proBNP than controls (Figure 1; Table 2). Six months after occlusion of ASD, the levels were still higher than in controls. Twelve months after intervention, patients no longer differed from controls (Figure 1; Table 2).

During the 12-month follow-up, the end-diastolic diameter of RV decreased after intervention (Table 3). The difference between patients and controls was significant at baseline and remained so up to 12 months after intervention (Figure 2; Table 3). The end-diastolic and systolic diameter and volume of LV increased after cessation of shunting through ASD (Table 3). At baseline, it was smaller than that of controls, with a median of -0.25 (range $-1.75-2.00$ ) SD $(P<.001)$ but equal to controls at the 6- and 12-month follow-ups. Similarly, the end-diastolic and systolic volumes of LV increased in 3D echocardiography during follow-up (Table 3).

4.2. Children with PDA. All 25 children with PDA underwent percutaneous closure. In 19 children, the PDA was occluded with a Flipper detachable coil (Cook, Bloomington, IN) and in 7 with an Amplatzer Duct Occluder (AGA Medical Corp., Golden Valley, MN). In these children, no other heart defects were observed. One child had a minimal residual shunt 6 months after coil occlusion. Children with PDA had higher levels of ANPN and NT-proBNP than controls before occlusion of PDA. After intervention, levels of both peptides decreased but were still somewhat higher than in controls at the 6-month follow-up (Figure 1, Table 2). The $\mathrm{LV}$ end-diastolic and systolic diameter and volume were higher in children with PDA than in controls at baseline, but no difference was seen 6 months after treatment (Figure 3; Table 3). The end-diastolic diameter of RV increased after occlusion of PDA (Table 3).

4.3. Children with CoA. Of the 15 children with CoA, 11 had native CoA and 4 recurrent CoA after surgery. Ten patients had a bicuspid aortic valve and one patient had mild mitral valve regurgitation. Five patients underwent balloon dilatation, one of them with a stent implantation. Ten patients were operated on with resection and end-toend anastomosis. At baseline, the arm-leg blood pressure gradient measured a median of 30 (range $20-56) \mathrm{mm} \mathrm{Hg}$. It decreased to a median of 3 (range $0-16) \mathrm{mm} \mathrm{Hg}(P=.001)$ at the 6-month follow-up. Systolic blood pressure measured a median of 120 (range 96-151) mm Hg in patient group and 101 (range $72-123) \mathrm{mm} \mathrm{Hg}$ in controls $(P<.001)$. During the 12-month follow-up, it decreased to a median of 105 (range $83-117) \mathrm{mm} \mathrm{Hg}$ in patients $(P=.001$ compared with baseline) and did not differ from that of controls.

At baseline, serum levels of ANPN were higher than in controls (Figure 1; Table 2). After relief of CoA, a significant reduction occurred in levels of peptides as compared with 


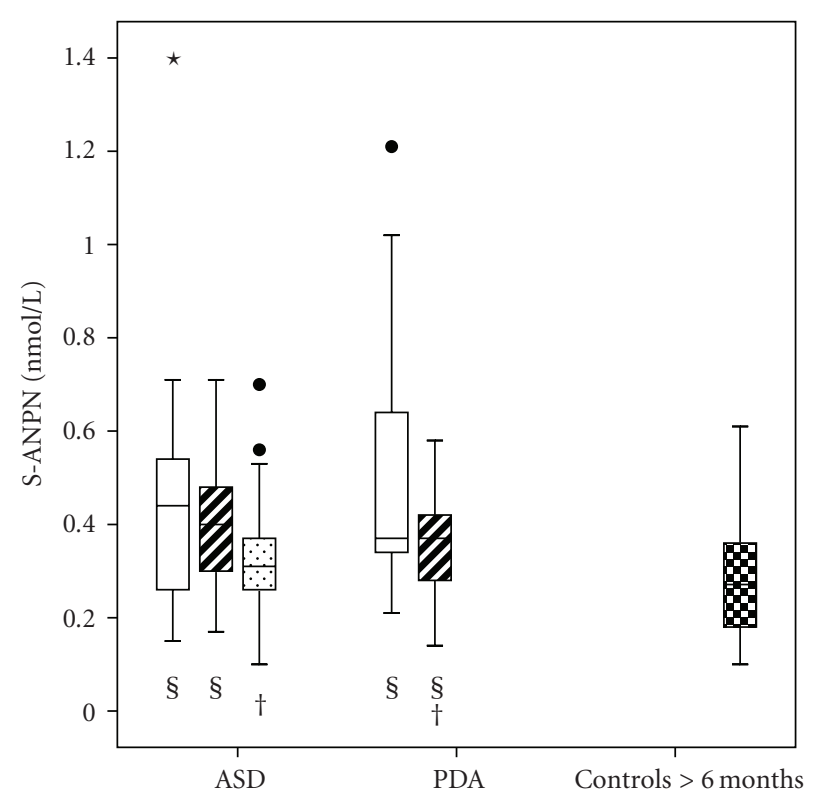

$\square$ Baseline

12 months

B Controls $>6$ months

(a)

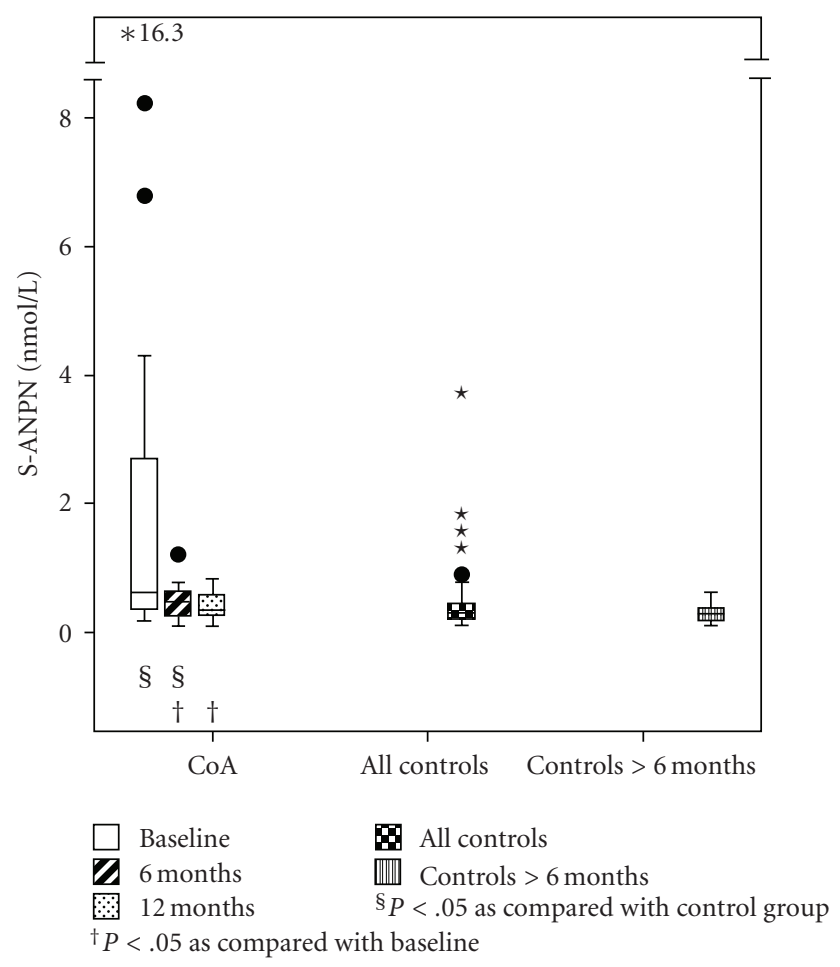

(c)

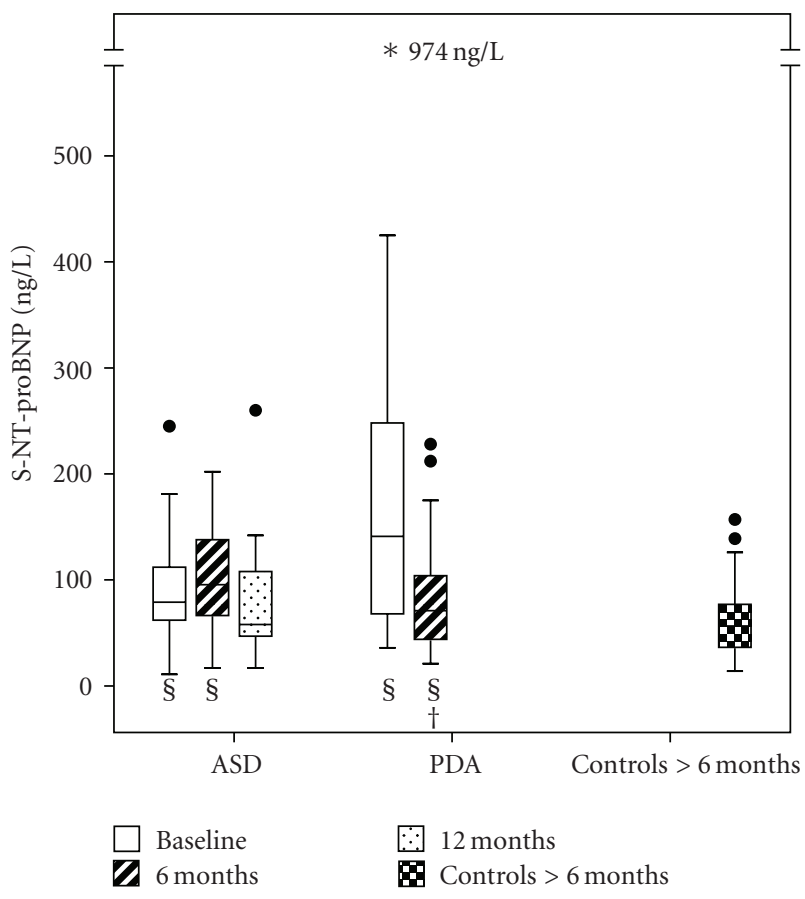

(b)

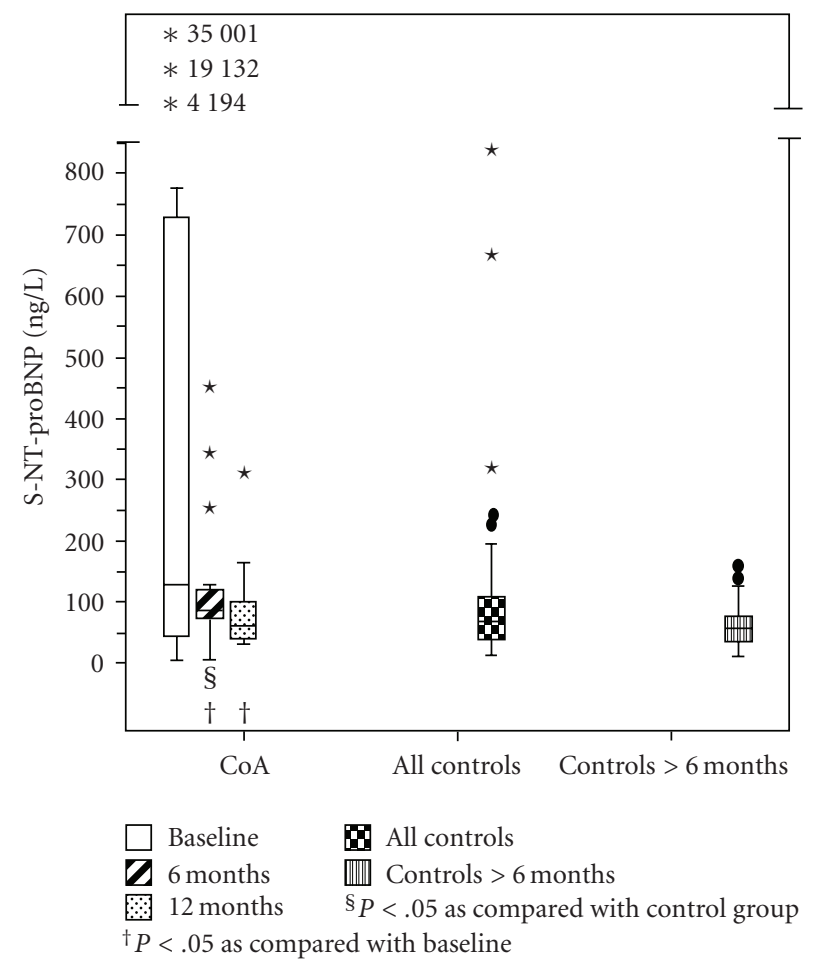

(d)

FIGURE 1: Serum levels of (a) N-terminal proatriopeptide (ANPN) and (b) N-terminal probrain natriuretic peptide (NT-proBNP) in patients with atrial septal defect (ASD) and patent ductus arteriosus (PDA) measured at baseline and at 6 and 12 months' follow-up, and in controls older than six months. Serum levels of (c) N-terminal proatriopeptide (ANPN) and (d) N-terminal probrain natriuretic peptide (NTproBNP) in patients with coarctation of the aorta measured at baseline and at 6 and 12 months' follow-up, and in all controls and controls older than six months. Whiskers at the top and bottom of each box indicate maximum and minimum; the top and bottom of each box the third and first quartiles; line through the box the median. Solid circles are outliers and $*$ indicates an extreme case. 
TABLE 2: Serum levels of ANPN and NT-proBNP in patients measured at baseline and at 6 and 12 months' follow-up, and in controls. Values are expressed as median (range).

\begin{tabular}{|c|c|c|c|}
\hline & $N$ & ANPN (nmol/L) & NT-proBNP (ng/L) \\
\hline & & Median (range) & Median (range) \\
\hline Controls & 76 & $0.30(0.10-3.70)$ & $68(14-3224)$ \\
\hline $\begin{array}{l}\text { Controls } \\
>0.5 \text { year }\end{array}$ & 64 & $0.28(0.10-0.61)$ & $57(14-157)$ \\
\hline $\begin{array}{l}\text { ASD } \\
\text { baseline }\end{array}$ & 21 & $0.44^{\S}(0.15-1.40)$ & $79^{\S}(11-245)$ \\
\hline $\begin{array}{l}\text { ASD } \\
6 \text { months }\end{array}$ & 20 & $0.40^{\S}(0.17-0.71)$ & $96^{\S}(17-202)$ \\
\hline $\begin{array}{l}\text { ASD } \\
1 \text { year }\end{array}$ & 21 & $0.31^{\dagger}(0.10-0.70)$ & $58(17-260)$ \\
\hline $\begin{array}{l}\text { PDA } \\
\text { baseline }\end{array}$ & 25 & $0.37^{\S}(0.21-1.21)$ & $141^{\S}(36-974)$ \\
\hline $\begin{array}{l}\text { PDA } \\
6 \text { months }\end{array}$ & 25 & $0.37^{\S \dagger}(0.14-0.58)$ & $71^{\S \dagger}(21-228)$ \\
\hline $\begin{array}{l}\text { CoA } \\
\text { baseline }\end{array}$ & 15 & $0.60^{\S}(0.16-16.30)$ & $128(5-35001)$ \\
\hline $\begin{array}{l}\text { CoA } \\
6 \text { months }\end{array}$ & 15 & $0.46^{\S \dagger}(0.09-1.20)$ & $86^{\S \dagger}(9-441)$ \\
\hline $\begin{array}{l}\text { CoA } \\
12 \text { months }\end{array}$ & 13 & $0.34^{\dagger}(0.09-0.82)$ & $60^{\dagger}(32-309)$ \\
\hline
\end{tabular}

ANPN: N-terminal proatriopeptide; ASD: atrial septal defect; CoA: coarctation of the aorta;

Mos: months; NT-proBNP: N-terminal probrain natriuretic peptide; PDA: patent ductus arteriosus;

$\S_{P}<.05$ as compared with control group $>0.5$ years except CoA group at baseline is compared with whole control group;

${ }^{\dagger} P<.05$ as compared with baseline.

baseline. At the 6-month follow-up, levels of both ANPN and NT-proBNP were higher than in controls but after one year, the levels were similar to controls (Table 2).

Six months after treatment, the $z$-score of the enddiastolic thickness of the interventricular septum had decreased, but it increased again by the 12-month follow-up (Figure 4). The end-diastolic diameter of RV increased during follow-up (Table 3). In 3D echocardiography, the enddiastolic and systolic volumes of LV remained unchanged.

4.4. Comparison of Patient Groups at Baseline. Among patients older than 6 months, levels of NT-proBNP were the highest in children with PDA $(P=.004$ as compared with patients with $\mathrm{CoA}, P=.032$ as compared with those with ASD), followed by those with ASD ( $P=$ ns as compared with patients with CoA), and finally those with CoA. ANPN levels at baseline were similar in all patient groups, but higher in these than in controls.

4.5. Correlations. At baseline, serum levels of ANPN and NT-proBNP correlated with 2D and 3D echocardiographic dimensions of RV and LV according to the loading condition. In the ASD group, serum levels of NT-proBNP correlated with the end-systolic volume of $\operatorname{LV}(r=0.487, P=.025)$. In patients with PDA and volume overload of the left side of the heart, serum levels of ANPN and pro-BNP correlated with LV end-diastolic diameter $(r=0.513, P=.009$, and $r=0.446, P=.025$, resp. $)$ and volume $(r=0.464, P=.020$ and $r=0.409, P=.042$, resp.) in 2D echocardiography. In patients with $\mathrm{CoA}$, negative correlations were found between levels of ANPN and NT-proBNP and 3D echocardiographic end-diastolic volume of LV $(r=-0.719, P=.003$; and $r=-0.744, P=.001$, resp.).

\section{Discussion}

In this prospective follow-up study, we evaluated the influence of treatment for different types of loading conditions on serum levels of ANPN and NT-proBNP, and correlated peptide levels to $2 \mathrm{D}$ and $3 \mathrm{D}$ echocardiographic findings. ANPN levels were higher than in controls at baseline in all patient groups, and NT-proBNP in patients with ASD and PDA. Both peptide levels were elevated 6 months after treatment, and decreased thereafter. At the 6-month followup, the patients with PDA were the only group in whom the dimensions of the originally overloaded ventricle had normalized. The end-diastolic diameter of RV was larger in patients with ASD than in controls and the interventricular septum thicker in patients with CoA than in controls.

Shunting through the ASD causes underfilling of the LV, and volume overload of the RV disturbs ventricular septal motion $[24,25]$. The diastolic and systolic function of the LV 
TABLE 3: Two- and three-dimensional echocardiographic findings in control subjects and in groups of children with ASD, PDA, and CoA, measured at baseline and at 6 and 12 months after repair. Values are given as median (range).

\begin{tabular}{|c|c|c|c|c|c|c|c|c|c|}
\hline \multirow[t]{2}{*}{ Diagnosis } & \multirow[t]{2}{*}{ s Controls } & \multicolumn{3}{|c|}{ ASD } & \multicolumn{2}{|c|}{ PDA } & \multicolumn{3}{|c|}{$\mathrm{CoA}$} \\
\hline & & & $\begin{array}{c}6 \text { months } \\
\mathrm{f}-\mathrm{u}\end{array}$ & $\begin{array}{c}12 \text { months } \\
\mathrm{f}-\mathrm{u}\end{array}$ & line & $\begin{array}{c}6 \text { months } \\
\mathrm{f}-\mathrm{u}\end{array}$ & line & $\begin{array}{c}6 \text { months } \\
\mathrm{f}-\mathrm{u}\end{array}$ & $\begin{array}{c}12 \text { months } \\
\mathrm{f}-\mathrm{u}\end{array}$ \\
\hline$N$ & 76 & 21 & & 21 & 2 & 25 & 15 & 15 & 13 \\
\hline IVSED & 0.00 & 0.25 & 0.00 & $0.50^{\dagger}$ & 0.50 & 0.25 & $1.25^{\S}$ & $0.50^{\dagger}$ & $1.50^{\S}$ \\
\hline (SD) & $(-2.00-2.75)$ & $(-1.00-3.75)$ & $(-2.00-1.25)$ & $(-1.50-2.50)$ & $(-1.75-4.50)$ & $(-1.75-4.25)$ & $(-0.75-5.00)$ & $(-1.25-2.50)$ & $(-0.00-2.75)$ \\
\hline LVPWED & -0.50 & -0.25 & -0.25 & $0.00^{\S \dagger}$ & -0.50 & -0.25 & $0.25^{\S}$ & 0.00 & $0.75^{\S}$ \\
\hline$(\mathrm{SD})$ & $(-2.25-2.00)$ & $(-1.50-2.00)$ & $(-2.25-1.00)$ & $(-1.25-1.25)$ & $(-2.00-2.75)$ & $(-1.25-2.25)$ & $(-2.50-1.75)$ & $(-1.50-1.50)$ & $(-0.75-1.75)$ \\
\hline RVEDD & 0.25 & $4.75^{\S}$ & $1.25^{\S \dagger}$ & $2.25^{\S \dagger}$ & 0.125 & $0.625^{\S \dagger}$ & -0.375 & $0.75^{\dagger}$ & 0.50 \\
\hline (SD) & $(-1.75-2.50)$ & $(2.25-9.75)$ & $(-1.50-5.50)$ & $(-0.25-3.50)$ & $(-1.75-2.75)$ & $(-1.75-3.50)$ & $(-2.25-1.75)$ & $(-2.00-2.00)$ & $(-2.25-1.75)$ \\
\hline LVEDD & -0.25 & $-1.50^{\S}$ & $0.00^{\dagger}$ & $-0.50^{\dagger}$ & $0.75^{\S}$ & $0.00^{\dagger}$ & $0.75^{\S}$ & $1.00^{\S}$ & $1.00^{\S}$ \\
\hline (SD) & $(-1.75-2.00)$ & $(-3.75-0.00)$ & $(-3.00-1.25)$ & $(-1.75-1.25)$ & $(-1.25-3.75)$ & $(-2.00-1.75)$ & $(-1.25-4.25)$ & $(-1.00-3.25)$ & $(-0.50-2.00)$ \\
\hline LVEDV & 62.1 & $47.2^{\S}$ & $63.0^{\dagger}$ & $60.4^{\dagger}$ & $78.4^{\S}$ & $64.2^{\dagger}$ & $75.0^{\S}$ & 65.8 & $72.5^{\S}$ \\
\hline$\left(\mathrm{mL} / \mathrm{m}^{2}\right)$ & $(36.9-87.7)$ & $(27.6-67.1)$ & $(35.5-83.1)$ & $(43.5-92.9)$ & $(44.7-117.1)$ & $(37.6-89.5)$ & $(47.9-115.6)$ & (49.8-98.5) & $(52.5-82.4)$ \\
\hline LVESV & 19.3 & $13.2^{\S}$ & $18.5^{\dagger}$ & $19.4^{\dagger}$ & $24.5^{\S}$ & $19.5^{\dagger}$ & 19.7 & 18.4 & 18.5 \\
\hline$\left(\mathrm{mL} / \mathrm{m}^{2}\right)$ & $(7.7-30.6)$ & $(7.4-33.2)$ & $(8.0-28.2)$ & $(12.5-31.8)$ & $(12.3-45.0)$ & $(11.9-33.3)$ & $(12.8-68.1)$ & $(8.9-37.4)$ & $(9.2-32.8)$ \\
\hline LVED3D & 56.2 & $49.2^{\S}$ & $63.5^{\S \dagger}$ & $65.1^{\S \dagger}$ & 57.5 & 57.7 & 64.7 & 61.4 & $71.4^{\S}$ \\
\hline$\left(\mathrm{mL} / \mathrm{m}^{2}\right)$ & $(32.0-89.5)$ & $(31.0-65.3)$ & $(36.5-93.8)$ & $(40.0-107.6)$ & $(43.6-99.4)$ & $(41.0-84.2)$ & (35.6-145.3) & $(30.7-131.1)$ & $(35.7-121.2)$ \\
\hline LVES3D & 28.5 & $24.1^{\S}$ & $31.0^{\dagger}$ & $33.8^{\S \dagger}$ & 29.8 & 27.6 & 38.1 & 28.8 & 30.2 \\
\hline$\left(\mathrm{mL} / \mathrm{m}^{2}\right)$ & $(14.1-52.8)$ & $(16.2-38.8)$ & $(17.5-45.7)$ & $(18.1-48.9)$ & $(19.4-54.1)$ & $(18.1-37.6)$ & $(16.8-93.5)$ & $(9.3-80.1)$ & $(14.9-56.5)$ \\
\hline
\end{tabular}

ASD: atrial septal defect, CoA: coarctation of the aorta; IVSED (SD): $z$-score (SD) of the end-diastolic thickness of interventricular septum; LVEDD (SD): $z$ score (SD) of the end-diastolic diameter of left ventricle; LVESD (SD): $z$-score (SD) of the end-systolic diameter of left ventricle; LVEDV: end-diastolic volume of left ventricle adjusted for body surface area; LVESV: end-systolic volume of left ventricle adjusted for body surface area; LVED3D: end-diastolic volume of left ventricle adjusted for body surface area measured by 3D echocardiography; LVES3D: end-systolic volume of left ventricle adjusted for body surface area measured by 3D echocardiography, LVPWED (SD): $z$-score (SD) of the end-diastolic thickness of left ventricular posterior wall; PDA: patent ductus arteriosus; RVEDD (SD): $z$-score (SD) of the end-diastolic diameter of right ventricle; SD: standard deviation of published normal values;

$\S_{P}<.05$ as compared with control group;

${ }^{\dagger} P<.05$ as compared with baseline.

can thus be mechanically disturbed [24]. Acute improvement in preload and early diastolic function of the LV after ASD closure depends on the degree of the RV volume overload and is associated with normalization of the ventricular septal motion and ventricular interdependence [26]. LV symmetry increases months after surgical ASD repair [27, 28].

In some studies, end-diastolic diameter of the RV has decreased after ASD closure, but remained enlarged as compared with that of controls [15, 29-32]. Some studies have demonstrated that after closure of the defect, enddiastolic diameters and volumes of LV and RV change towards normal values $[33,34]$. Similarly, in our patients, size of LV increased during follow-up, and no difference was present between patients and controls 6 months after treatment. The end-diastolic diameter of RV decreased, but remained larger than in controls up to 12 months after treatment [35].

In pediatric patients with ASD, blood levels of ANPN have been shown to correlate with the degree of shunting [36]. After closure of ASD, levels of ANPN and volume of RV have decreased [13]. Serum levels of ANPN have been higher than in matched controls up to 35 years after surgical closure of ASD [37]. On the other hand, plasma levels of ANP and BNP have normalized during a 3month follow-up after device closure of ASD in children
[15]. In our study, six months after ASD closure, the difference between patients and controls in serum levels of ANPN and NT-proBNP was still evident, but not after 12 months. Closure of ASD redirects blood flow to LV. Therefore, in our study, the size of LV increased. This may be why at 6 months the peptide levels had not started to decrease.

We have earlier shown that a day after PDA occlusion, serum levels of NT-proBNP increase, but decrease to control levels in 6 months [23]. Our finding of the highest levels of NT-proBNP in patients with PDA and volume overload of $\mathrm{LV}$ is in accordance with the previous studies $[38,39]$. To our knowledge, no correlation between echo parameters and peptide levels has been published in patients with left-sided volume overload caused by PDA. In our patients with PDA, a significant correlation was found between serum levels of ANPN and NT-proBNP and the end-diastolic diameter and volume of $\mathrm{LV}$.

Despite early and apparently successful repair of CoA, myocardial hypertrophy may persist, even in the presence of LV remodeling [40-43]. In our study, after treatment for CoA, the thickness of the interventricular septum after the first 6 months decreased and the diameter of RV increased [44]. At baseline, children with CoA had higher levels of ANPN than controls, but the difference in levels 


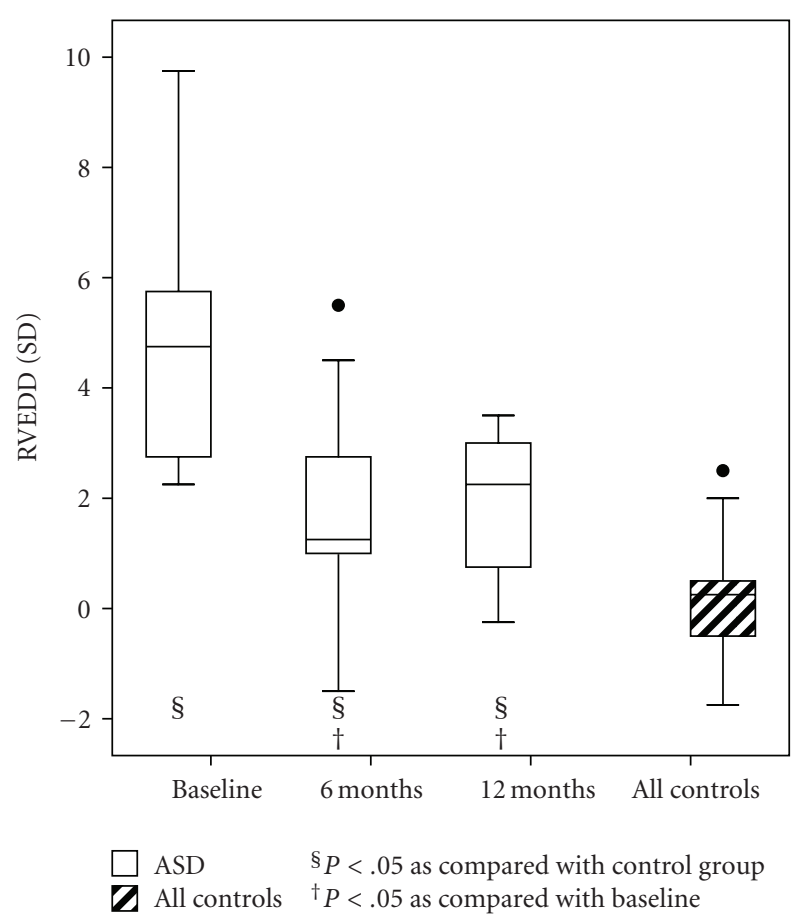

Figure 2: The $z$-score (SD) of end-diastolic diameter of right ventricle (RVEDD) in patients with atrial septal defect (ASD) and in controls. Whiskers at the top and bottom of each box indicate maximum and minimum; the top and bottom of each box the third and first quartiles; line through the box the median. Solid circles are outliers.

of NT-proBNP failed to reach statistical significance. The difference between patients and controls in levels of both peptides was evident at the 6-month follow-up. Serum levels of ANPN and NT-proBNP correlated negatively with size of LV in 3D echocardiography. This may reflect diastolic ventricular dysfunction and restrictive physiology of the ventricles even after repair, leading to dilatation of atria.

When different patient groups were compared at baseline, no differences emerged in levels of ANPN and NTproBNP between volume and pressure overload of LV. Volume overload of LV caused higher levels of NT-proBNP than that of RV, but no differences in levels of ANPN were seen. Others have found higher plasma levels of ANP in volume overload of LV (VSD and PDA) than in right- sided volume overload [12]. In our patients with ASD and CoA, no difference was evident. The effects of volume overload on the levels of ANPN and BNP have been investigated in patients with VSD and PDA, but no follow-up studies are available. Plasma levels of BNP have been demonstrated to correlate with systolic RV pressure in children with volume overload of RV [45].

Plasma levels of BNP have been shown to correlate with right atrial and ventricular pressures in a child population consisting of different loading conditions and a wide age range [14]. In adults, plasma levels of BNP are higher

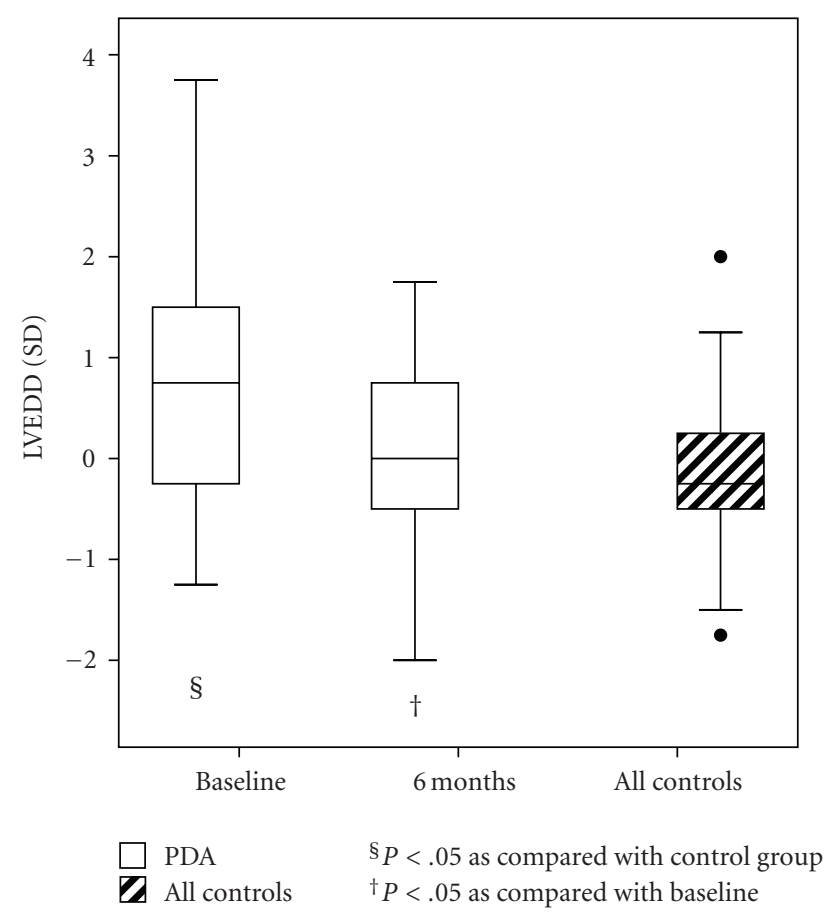

Figure 3: The $z$-score (SD) of end-diastolic diameter of left ventricle (LVEDD) in patients with patent ductus arteriosus (PDA) and in controls. Whiskers at the top and bottom of each box indicate maximum and minimum; the top and bottom of each box the third and first quartiles; line through the box the median. Solid circles are outliers.

in patients with acquired LV dysfunction. They are also higher in those with congenital heart defect and RV failure than in patients without structural heart disease [46]. ANP and BNP may have different regulation of gene expression depending on the form and localization of the stress exerted on the heart [47]. This may explain why in our study the correlation between serum levels of natriuretic peptides and echocardiographic dimensions varied under different loading conditions.

The most significant limitation to this study is that the number of patients in each group of patients is small and therefore we cannot compare the patients undergoing surgical and percutanoeus treatment. The age range is wide in patient groups and controls. The controls were examined only once. However, we assumed that the levels of peptides would not change significantly during one year.

In conclusion, serum levels of both ANPN and NTproBNP are higher in patients with ASD or PDA than in controls. In patients with CoA, ANPN levels are higher than in controls. After treatment, peptide levels decrease and some remodeling of the originally loaded ventricle takes place during within 6-12 months. Natriuretic peptide levels correlate with echocardiographic findings and reflect the hemodynamic loading condition. Therefore, natriuretic peptides might be useful in the pre-and postoperative followup of these patient groups. 


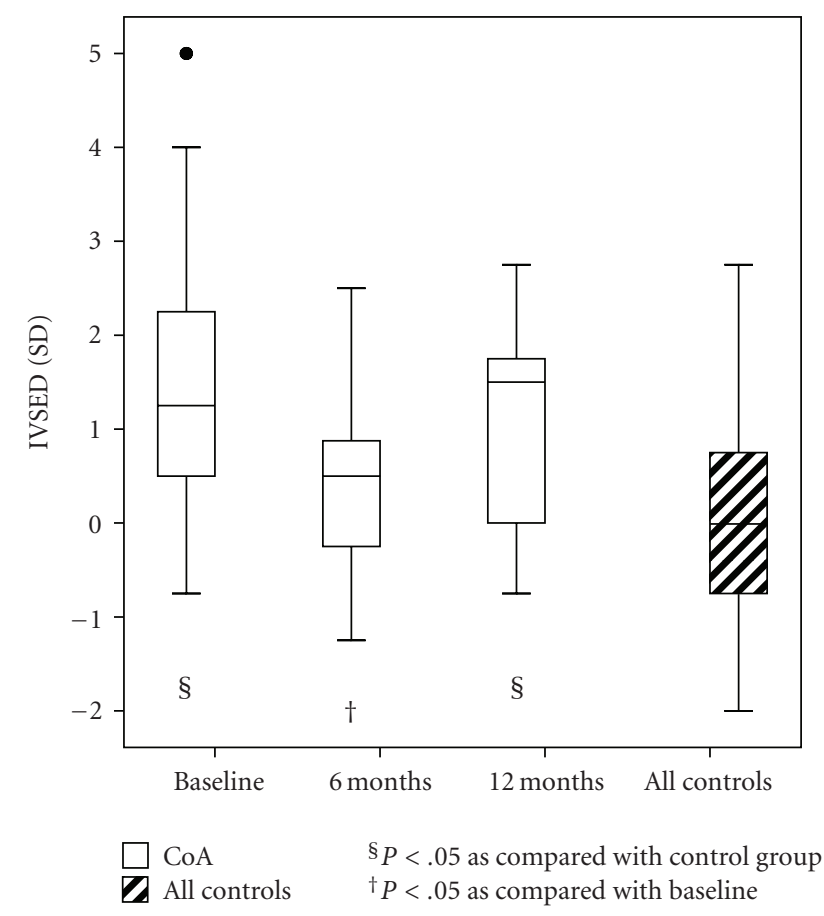

FIgURE 4: The $z$-score (SD) of end-diastolic thickness of interventricular septum (IVSED) in patients with coarctation of the aorta (CoA) and in controls. Whiskers at the top and bottom of each box indicate maximum and minimum; the top and bottom of each box the third and first quartiles; line through the box the median. Solid circle is an outlier.

\section{Acknowledgments}

This study was supported by grants from the Finnish Foundation for Cardiovascular Research, the Foundation for Pediatric Research, and the special governmental subsidy for health sciences research, all in Helsinki, Finland.

\section{References}

[1] J. P. Goetze, "ProBNP-derived peptides in cardiac disease," Scandinavian Journal of Clinical and Laboratory Investigation, vol. 64 , no. 5, pp. 497-510, 2004.

[2] L. B. Cea, "Natriuretic peptide family: new aspects," Current Medicinal Chemistry: Cardiovascular and Hematological Agents, vol. 3, no. 2, pp. 87-98, 2005.

[3] V. K. Munagala, J. C. Burnett Jr., and M. M. Redfield, "The natriuretic peptides in cardiovascular medicine," Current Problems in Cardiology, vol. 29, no. 12, pp. 707-769, 2004.

[4] A. J. de Bold, K. K. Ma, Y. Zhang, M. L. de Bold, M. Bensimon, and A. Khoshbaten, "The physiological and pathophysiological modulation of the endocrine function of the heart," Canadian Journal of Physiology and Pharmacology, vol. 79, no. 8, pp. 705-714, 2001.

[5] C. Hall, "NT-ProBNP: the mechanism behind the marker," Journal of Cardiac Failure, vol. 11, no. 5, supplement, pp. S81S83, 2005.

[6] A. Koch and H. Singer, "Normal values of B type natriuretic peptide in infants, children, and adolescents," Heart, vol. 89, no. 8, pp. 875-878, 2003.
[7] M. Yoshibayashi, T. Kamiya, Y. Saito, et al., "Plasma brain natriuretic peptide concentrations in healthy children from birth to adolescence: marked and rapid increase after birth," European Journal of Endocrinology, vol. 133, no. 2, pp. 207209, 1995.

[8] T. S. Mir, R. Laux, H. H. Hellwege, et al., "Plasma concentrations of aminoterminal pro atrial natriuretic peptide and aminoterminal pro brain natriuretic peptide in healthy neonates: marked and rapid increase after birth," Pediatrics, vol. 112, no. 4, pp. 896-899, 2003.

[9] M. Rauh and A. Koch, "Plasma N-terminal pro-B-type natriuretic peptide concentrations in a control population of infants and children [10]," Clinical Chemistry, vol. 49, no. 9, pp. 1563-1564, 2003.

[10] A. Nir, B. Bar-Oz, Z. Perles, R. Brooks, A. Korach, and A. J. Rein, "N-terminal pro-B-type natriuretic peptide: reference plasma levels from birth to adolescence. Elevated levels at birth and in infants and children with heart diseases," Acta Paediatrica, vol. 93, no. 5, pp. 603-607, 2004.

[11] J. Weil, F. Bidlingmaier, C. Dohlemann, U. Kuhnle, T. Strom, and R. E. Lang, "Comparison of plasma atrial natriuretic peptide levels in healthy children from birth to adolescence and in children with cardiac diseases," Pediatric Research, vol. 20, no. 12, pp. 1328-1331, 1986.

[12] S. Matsuoka, Y. Kurahashi, Y. Miki, et al., "Plasma atrial natriuretic peptide in patients with congenital heart diseases," Pediatrics, vol. 82, no. 4, pp. 639-643, 1988.

[13] A. R. Erbay, M. B. Yilmaz, M. Balci, and I. Sabah, "Atrial natriuretic peptide levels in adult patients before and after surgery for correction of atrial septal defects: relationship with atrial arrhythmias," Clinical Science, vol. 107, no. 3, pp. 297302, 2004.

[14] C. G. Cowley, J. D. Bradley, and R. E. Shaddy, "B-type natriuretic peptide levels in congenital heart disease," Pediatric Cardiology, vol. 25, no. 4, pp. 336-340, 2004.

[15] H. Muta, M. Ishii, Y. Maeno, T. Akagi, and H. Kato, "Quantitative evaluation of the changes in plasma concentrations of cardiac natriuretic peptide before and after transcatheter closure of atrial septal defect," Acta Paediatrica, vol. 91, no. 6, pp. 649-652, 2002.

[16] B. Zeevi, I. Gil-Ad, R. Zabreski, et al., "Interventional catheterization decreases plasma levels of atrial natriuretic peptide (ANP) in children with congenital heart defects," Catheterization and Cardiovascular Diagnosis, vol. 45, no. 1, pp. 27-32, 1998.

[17] K. M. Borow and R. Karp, "Atrial septal defect-lessons from the past, directions for the future," The New England Journal of Medicine, vol. 323, no. 24, pp. 1698-1700, 1990.

[18] M. Campbell, "Natural history of atrial septal defect," British Heart Journal, vol. 32, no. 6, pp. 820-826, 1970.

[19] M. Samanek, "Children with congenital heart disease: probability of natural survival," Pediatric Cardiology, vol. 13, no. 3, pp. 152-158, 1992.

[20] L. Benson and P. McLaughlin, "Coarctation of the aorta," in The Natural and Modified History of Congenital Heart Disease, R. Freedom, S. J. Yoo, H. Mikailian, and W. G. Williams, Eds., vol. 1, pp. 251-275, Blackwell, New York, NY, USA, 2004.

[21] A. Rothman, "Coarctation of the aorta: an update," Current Problems in Pediatric and Adolescent Health Care, vol. 28, no. 2, pp. 33-60, 1998.

[22] C. Kampmann, C. M. Wiethoff, A. Wenzel, et al., "Normal values of $\mathrm{M}$ mode echocardiographic measurements of more than 2000 healthy infants and children in central Europe," Heart, vol. 83, no. 6, pp. 667-672, 2000. 
[23] A. Eerola, E. Jokinen, T. Boldt, and J. Pihkala, "The influence of percutaneous closure of patent ductus arteriosus on left ventricular size and function: a prospective study using twoand three-dimensional echocardiography and measurements of serum natriuretic peptides," Journal of the American College of Cardiology, vol. 47, no. 5, pp. 1060-1066, 2006.

[24] R. O. Bonow, J. S. Borer, D. R. Rosing, S. L. Bacharach, M. V. Green, and K. M. Kent, "Left ventricular functional reserve in adult patients with atrial septal defect: pre- and postoperative studies," Circulation, vol. 63, no. 6, pp. 1315-1322, 1981.

[25] K. A. Popio, R. Gorlin, L. E. Teichholz, P. F. Cohn, D. Bechtel, and M. V. Herman, "Abnormalities of left ventricular function and geometry in adults with an atrial septal defect. Ventriculographic, hemodynamic and echocardiographic studies," American Journal of Cardiology, vol. 36, no. 3, pp. 302-308, 1975.

[26] A. Giardini, P. Moore, M. Brook, V. Stratton, and T. Tacy, "Effect of transcatheter atrial septal defect closure in children on left ventricular diastolic function," American Journal of Cardiology, vol. 95, no. 10, pp. 1255-1257, 2005.

[27] J. P. Hart, S. E. Cabreriza, R. F. Walsh, et al., "Echocardiographic analysis of ventricular geometry and function during repair of congenital septal defects," Annals of Thoracic Surgery, vol. 77, no. 1, pp. 53-60, 2004.

[28] R. E. Walker, A. M. Moran, K. Gauvreau, and S. D. Colan, "Evidence of adverse ventricular interdependence in patients with atrial septal defects," American Journal of Cardiology, vol. 93, no. 11, pp. 1374-1377, 2004.

[29] C. A. Pedra, J. Pihkala, K. J. Lee, et al., “Transcatheter closure of atrial septal defects using the Cardio-Seal implant," Heart, vol. 84, no. 3, pp. 320-326, 2000.

[30] G. R. Veldtman, V. Razack, S. Siu, et al., "Right ventricular form and function after percutaneous atrial septal defect device closure," Journal of the American College of Cardiology, vol. 37, no. 8, pp. 2108-2113, 2001.

[31] Z. D. Du, Q. L. Cao, P. Koenig, M. Heitschmidt, and Z. M. Hijazi, "Speed of normalization of right ventricular volume overload after transcatheter closure of atrial septal defect in children and adults," American Journal of Cardiology, vol. 88, no. 12, pp. 1450-1453, 2001.

[32] R. A. Meyer, J. C. Korfhagen, W. Covitz, and S. Kaplan, "Longterm follow-up study after closure of secundum atrial septal defect in children: an echocardiographic study," American Journal of Cardiology, vol. 50, no. 1, pp. 143-148, 1982.

[33] M. Nakazawa, J. M. Jarmakani, M. T. Gyepes, J. V. Prochazka, S. M. Yabek, and R. A. Marks, "Pre and postoperative ventricular function in infants and children with right ventricular volume overload," Circulation, vol. 55, no. 3, pp. 479-484, 1977.

[34] K. L. Wanderman, I. Ovsyscher, and M. Gueron, "Left ventricular performance in patients with atrial septal defect: evaluation with noninvasive methods," American Journal of Cardiology, vol. 41, no. 3, pp. 487-493, 1978.

[35] A. Eerola, J. I. Pihkala, T. Boldt, I. P. Mattila, T. Poutanen, and E. Jokinen, "Hemodynamic improvement is faster after percutaneous ASD closure than after surgery," Catheterization and Cardiovascular Interventions, vol. 69, no. 3, pp. 432-441, 2007.

[36] K. Kikuchi, K. Nishioka, T. Ueda, et al., "Relationship between plasma atrial natriuretic polypeptide concentration and hemodynamic measurements in children with congenital heart disease," Journal of Pediatrics, vol. 111, no. 3, pp. 335$342,1987$.
[37] T. E. Iivainen, K. W. Groundstroem, J. T. Lahtela, T. J. Talvensaari, A. Pasternack, and A. Uusitalo, "Serum Nterminal atrial natriuretic peptide in adult patients late after surgical repair of atrial septal defect," European Journal of Heart Failure, vol. 2, no. 2, pp. 161-165, 2000.

[38] D. Holmgren, A. Westerlind, P. A. Lundberg, and $H$. Wåhlander, "Increased plasma levels of natriuretic peptide type B and A in children with congenital heart defects with left compared with right ventricular volume overload or pressure overload," Clinical Physiology and Functional Imaging, vol. 25, no. 5, pp. 263-269, 2005.

[39] A. Westerlind, H. Wåhlander, G. Lindstedt, P. A. Lundberg, and D. Holmgren, "Clinical signs of heart failure are associated with increased levels of natriuretic peptide types B and A in children with congenital heart defects or cardiomyopathy," Acta Paediatrica, vol. 93, no. 3, pp. 340-345, 2004.

[40] M. C. Johnson, F. R. Gutierrez, D. R. Sekarski, C. M. Ong, and C. E. Canter, "Comparison of ventricular mass and function in early versus late repair of coarctation of the aorta," American Journal of Cardiology, vol. 73, no. 9, pp. 698-701, 1994.

[41] T. R. Kimball, J. M. Reynolds, W. A. Mays, P. Khoury, R. P. Claytor, and S. R. Daniels, "Persistent hyperdynamic cardiovascular state at rest and during exercise in children after successful repair of coarctation of the aorta," Journal of the American College of Cardiology, vol. 24, no. 1, pp. 194-200, 1994.

[42] L. Ý Sigurdardóttir and H. Helgason, "Echocardiographic evaluation of systolic and diastolic function in postoperative coarctation patients," Pediatric Cardiology, vol. 18, no. 2, pp. 96-100, 1997.

[43] G. Pacileo, G. Pacileo, C. Pisacane, et al., "Left ventricular remodeling and mechanics after successful repair of aortic coarctation," American Journal of Cardiology, vol. 87, no. 6, pp. 748-752, 2001.

[44] A. Eerola, A. Eerola, A. Eerola, et al., "Left ventricular hypertrophy persists after successful treatment for coarctation of the aorta," Scandinavian Cardiovascular Journal, vol. 41, no. 6, pp. 370-377, 2007.

[45] T. S. Mir, J. Falkenberg, B. Friedrich, et al., "Levels of brain natriuretic peptide in children with right ventricular overload due to congenital cardiac disease," Cardiology in the Young, vol. 15, no. 4, pp. 396-401, 2005.

[46] W. M. Book, B. J. Hott, and M. McConnell, "B-type natriuretic peptide levels in adults with congenital heart disease and right ventricular failure," American Journal of Cardiology, vol. 95, no. 4, pp. 545-546, 2005.

[47] N. Dzimiri, N. Dzimiri, N. Dzimiri, et al., "Differential regulation of atrial and brain natriuretic peptides and its implications for the management of left ventricular volume overload," European Journal of Clinical Investigation, vol. 32, no. 8, pp. 563-569, 2002. 


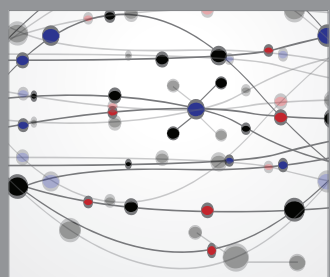

The Scientific World Journal
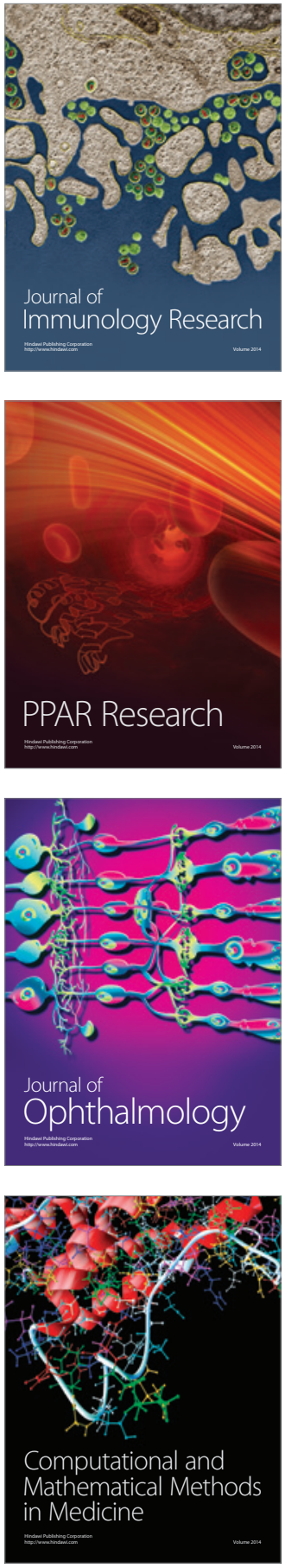

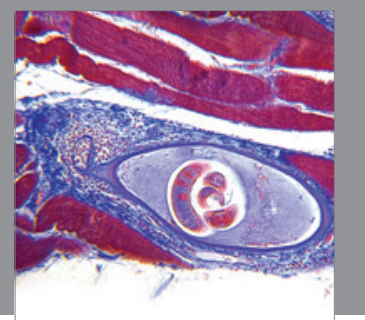

Gastroenterology

Research and Practice
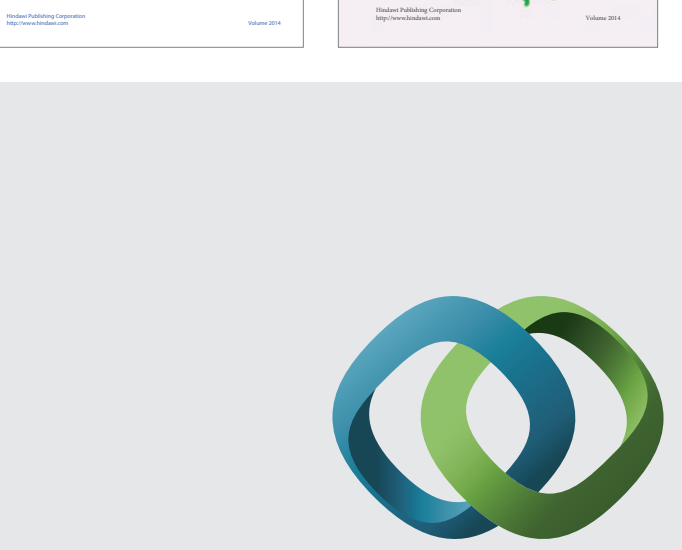

\section{Hindawi}

Submit your manuscripts at

http://www.hindawi.com
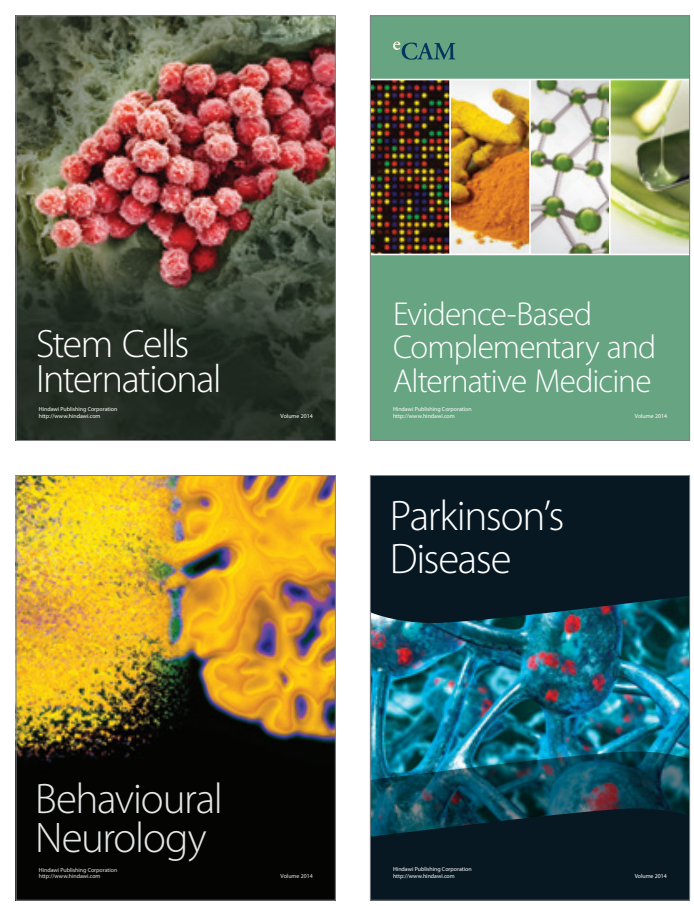

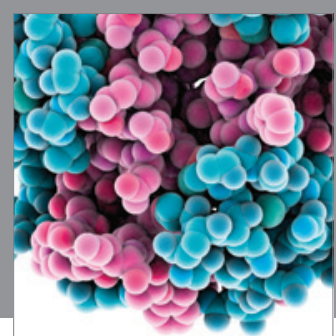

Journal of
Diabetes Research

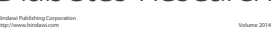

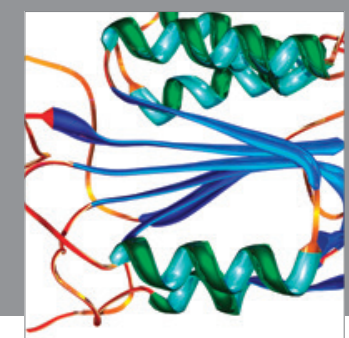

Disease Markers
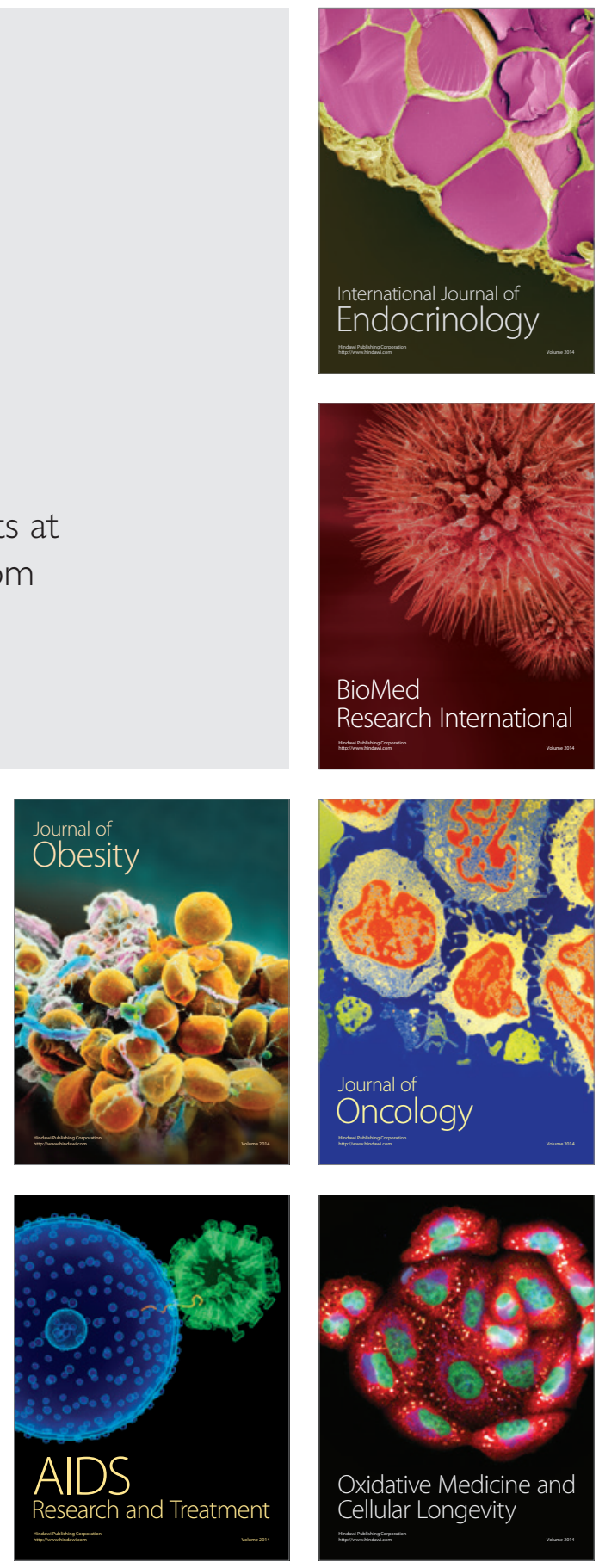